\title{
Adaptive Finite Element Strategies Based on Error Assessment
}

\author{
A. Huerta \\ B. A. Rodríguez-Ferran \\ C. P. Díez
}




\title{
Adaptive finite element strategies based on error assessment
}

\author{
Antonio Huerta, Antonio Rodríguez-Ferran and Pedro Díez
}

Departament de Matemàtica Aplicada III, E.T.S. de Ingenieros de Caminos

Edifici C2, Campus Nord, Universitat Politècnica de Catalunya

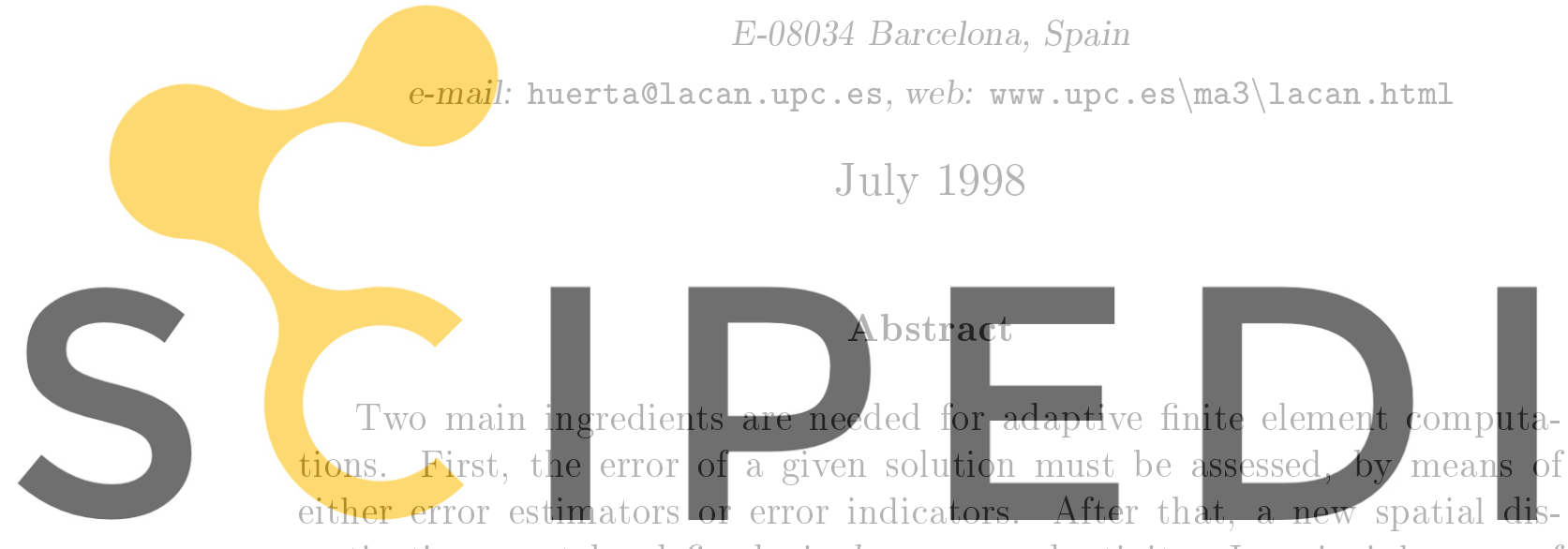

cretization must be defined via $h, p$ or $r$-adaptivity. In principle, any of

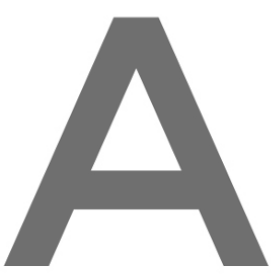

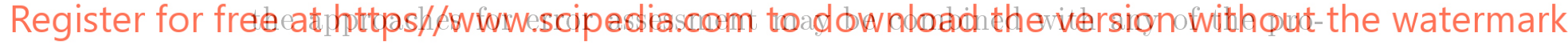

cedures for adapting the discretization. However, some combinations are clearly preferable. The advantages and limitations of the various alternatives are discussed. The most adequate strategies are illustrated by means of several applications in solid mechanics.

Keywords: adaptivity; error estimators; error indicators; nonlinear finite element analysis

Contract grant sponsor: Ministerio de Educación y Cultura; Contract grant number: TAP98-0421 


\section{Introduction}

Adaptive strategies are nowadays considered a standard tool in practical finite element computations. For any problem, adaptivity is an essential tool to obtain numerical solutions with a controlled accuracy. For some problems (typically in the nonlinear domain), adaptive strategies are even more fundamental: without them, a finite element solution simply cannot be computed. This is the case, for instance, with problems in nonlinear solid mechanics involving large strains or localization.

The two main ingredients of an adaptive procedure are 1) a tool for assessing the error of the solution computed with a given mesh and 2) an algorithm to define a new spatial discretization.

Two different approaches may be used for assessing the error: error estimators or error indicators. Error estimators approximate a measure of the actual error in a given norm. In this paper, the term error estimator means that the estimated error can be arbitrarily close to the true error. Other definitions are also standard; in some works $[1,2,3]$, error estimators are required to behave as equivalent norms of the actual error. Error indicators,
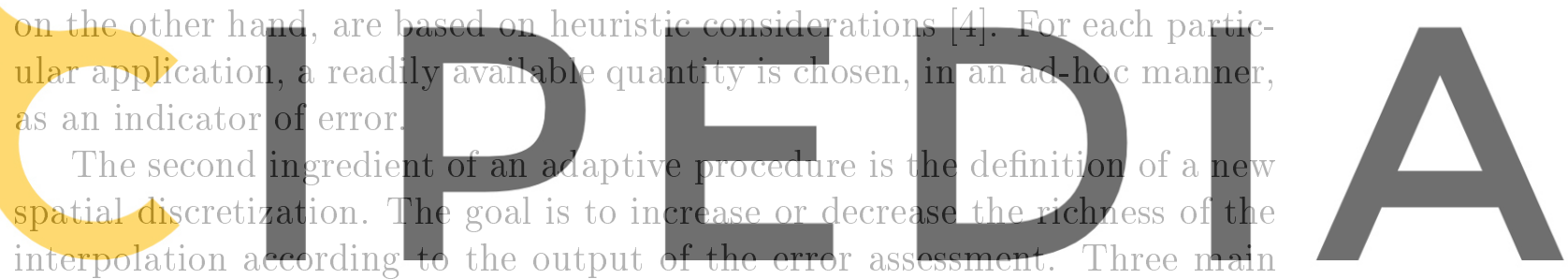

types of strategies may be used: $h$-adaptivity, $p$-adaptivity and $r$-adaptivity.

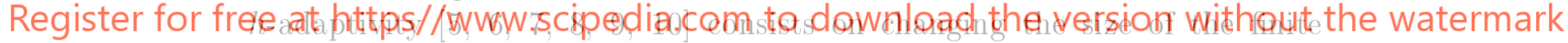

elements. In $p$-adaptivity, the degree of the interpolating polynomials is increased $[11,12,13]$. $r$-adaptivity consists on relocating the nodes, without changing the mesh connectivity $[14,15]$.

Various adaptive strategies can be devised by combining these ingredients. Consider, for instance, the necking test of Figure 1. This is a classical benchmark test in nonlinear computational mechanics [16]. A cylindrical bar is subjected to uniaxial extension. A slight geometric imperfection induces necking in the central part of the bar. If a coarse fixed mesh (i.e., no adaptivity) is employed, the result of Figure 1(a) is obtained (only one-fourth of the piece is shown). The elements in the neck zone become very distorted, following the large material deformation. As a consequence, a poor definition of the deformed shape of the piece is obtained. Two different adaptive strategies have been used to remedy this situation: $r$-adaptivity based on an error indicator and $h$-adaptivity based on an error estimator.

Figure 1(b) has been obtained by combining an $r$-adaptive technique (the 
arbitrary Lagrangian-Eulerian formulation) with a very simple error indicator: the element aspect ratio [17]. The nodes of the original coarse mesh are relocated to reduce the element distortion, and this enables a proper description of neck. Figure 1(c), on the other hand, is obtained by combining $h$-adaptivity and an error estimator [18]. A global accuracy of $2 \%$ is prescribed. This simple example illustrates the main features of the two strategies employed: the first one is simple and inexpensive, but there is no objective control on the accuracy; the second one is much more computationally involved, but a solution with a prescribed accuracy is obtained.

The goal of this paper is to discuss the advantages and limitations of various adaptive strategies, see section 2. The discussion focuses on the pros and cons of the various combinations of error assessment (indicators, estimators) and new spatial discretization ( $h, p, r$-adaptivity). The capabilities of adaptive strategies are illustrated in section 3 ( $r$-adaptivity with error indicators) and section 4 ( $h$-adaptivity with error estimation). Finally some concluding remarks are made in section 5 .
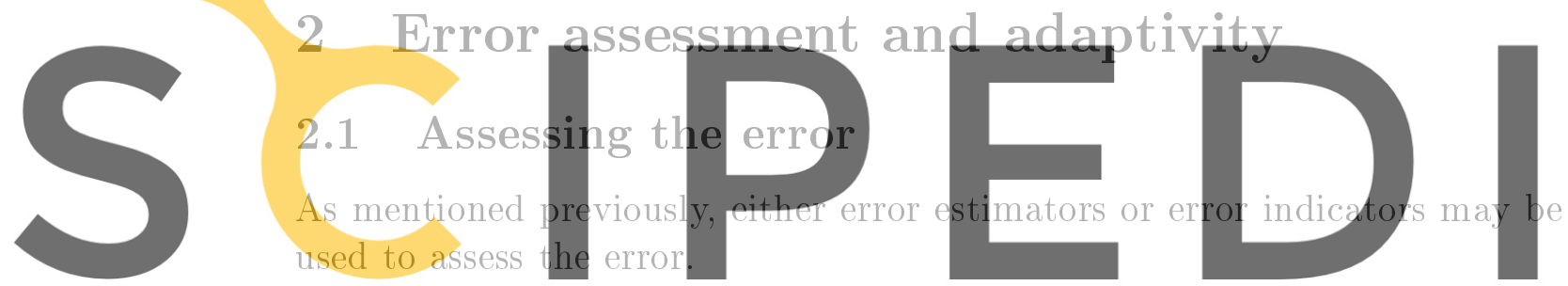

Error estimators may be classified into two groups: flux projection (ZZ-

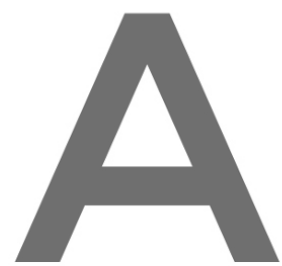

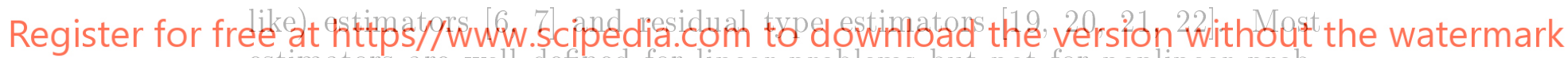

estimators are well defined for linear problems but not for nonlinear prob-

lems. For instance, the popular ZZ error estimator for linear problems is only an error indicator for nonlinear problems, because it is based on superconvergence properties that cannot be automatically extended to the nonlinear regime.

Here the estimator presented in detail in reference $[23,24,25]$ is employed. This estimator has a sound theoretical basis for both linear and nonlinear applications [18].

Various choices of an error indicator can be found in the literature. From a geometrical point of view, for instance, the element aspect ratio or, more generally, the distortion can be used [26]. In nonlinear solid mechanics, some common choices are the equivalent plastic (or, more generally, inelastic) strain or its gradient [4].

The advantages and limitations of error estimators and error indicators are summarized in Table 1. Error indicators are attractive because of their 
simplicity: they are based on very simple intuitive considerations (geometrical, mechanical, etc.) and can be computed easily and efficiently. Quantities used as error indicators are always readily available in the finite element computation, so the overhead cost is minimum. The drawback is that they are heuristic: the judgement of the user for defining a proper error indicator for a given problem is critical. Of course, error indicators are very specific of each particular application, and they must be calibrated (with the help of either analytical solutions in simple tests or error estimators). Moreover, error indicators only give relative information. Since the error is not quantified, an error indicator only tells where the spatial discretization must be richer, but not how much richer should it be.

Error estimators, on the other hand, must be based on firm mathematical foundations and are usually more expensive to evaluate than error indicators. In exchange for that, they have a major advantage: they provide an objective and quantitative information about the error. Moreover, the range of applicability of a certain error estimator is larger than for a given error indicator. The error estimator presented in reference [24, 18], for instance, is valid for any linear or nonlinear elliptic problem.
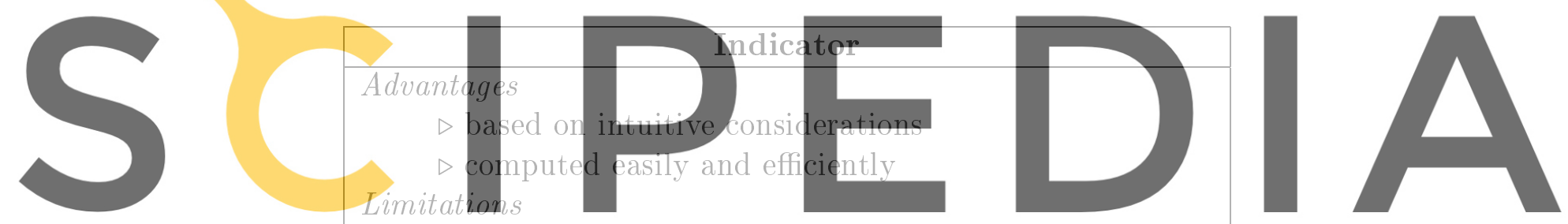

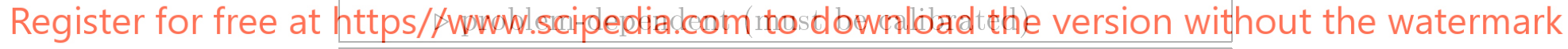

\begin{tabular}{|l|}
\hline Estimator \\
\hline Advantages \\
$\quad \triangleright$ objective measure of the actual error \\
$\quad$ wide range of applications \\
Limitations \\
$\triangleright$ need mathematical basis \\
$\triangleright$ usually more expensive to evaluate \\
\hline
\end{tabular}

Table 1: Comparison of error estimators and error indicators

\subsection{Adapting the spatial discretization}

Three strategies may be used to adapt the spatial discretization according to the error assessment: $h, p$ and $r$-adaptivity. $h$-adaptivity consists of build- 
ing a new mesh, using the same type of elements, and adapting the element size to the requirements of the solution. That is, reducing their size where the interpolation must be enriched (i.e. more accuracy is needed) and enlarging the elements where it is already accurate enough. The idea of $p$-adaptivity is to increase the order of the polynomials where a richer interpolation is needed, and maintain polynomials of low order where it is already rich enough. $r$-adaptivity consists on relocating the nodes to adapt the mesh to the requirements of the solution. The number of nodes and the mesh connectivity remain constant. Nodes are concentrated in zones where they are most needed. The mesh is allowed to coarsen in other parts of the domain, where a poorer interpolation suffices.

The merits and drawbacks of these three approaches are summarized in Table 2. $r$-adaptivity is easy to implement and inexpensive, because only the initial mesh is needed. Simple algorithms may be used to relocate the nodes. The transport of the information from the old mesh to the new mesh can be performed in a very natural way (by solving a convection equation), because these two meshes have the same connectivity. This intrinsic simplicity is also the cause of the limitations of $r$-adaptivity. The accuracy which can
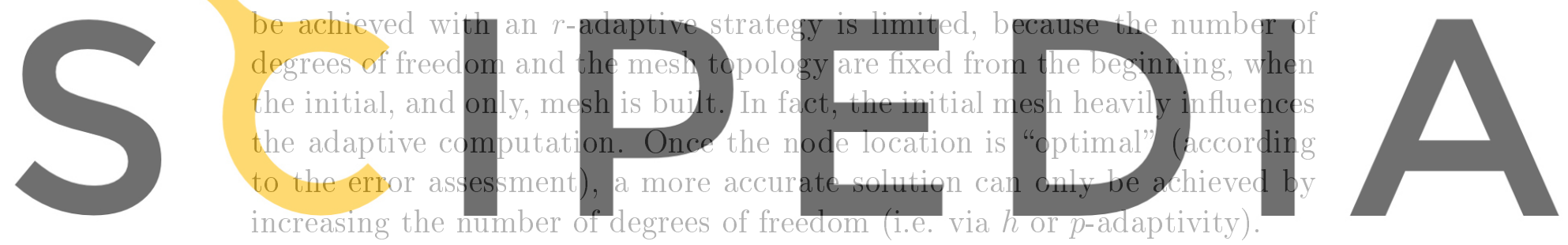

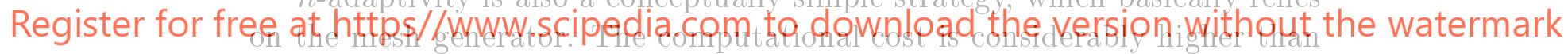

for $r$-adaptivity, because a new mesh must be generated at each step. After that, there are two alternatives: either restart the computation from scratch or project all the information from the old mesh to the new mesh. This transport is quite more involved than for $r$-adaptivity, because the two meshes may have very different topologies and numbers of elements. In exchange for this high cost, $h$-adaptivity is a very general approach: the number of degrees of freedom can change arbitrarily to meet a prescribed accuracy, and the initial mesh does not drastically influence the adaptive process, because a new mesh is rebuilt at each step.

From a theoretical standpoint, $p$-adaptivity has the advantage that it provides the fastest rate of convergence as the number of degrees of freedom increases. Moreover, it is the only strategy that can reach very high accuracies. However, the implementation is tedious: special care is needed to match two adjacent elements of different order. Moreover, this strategy is heavily dependent on the initial mesh. In practice, $p$-adaptivity is typically 
combined with $h$-adaptivity [12, 27].

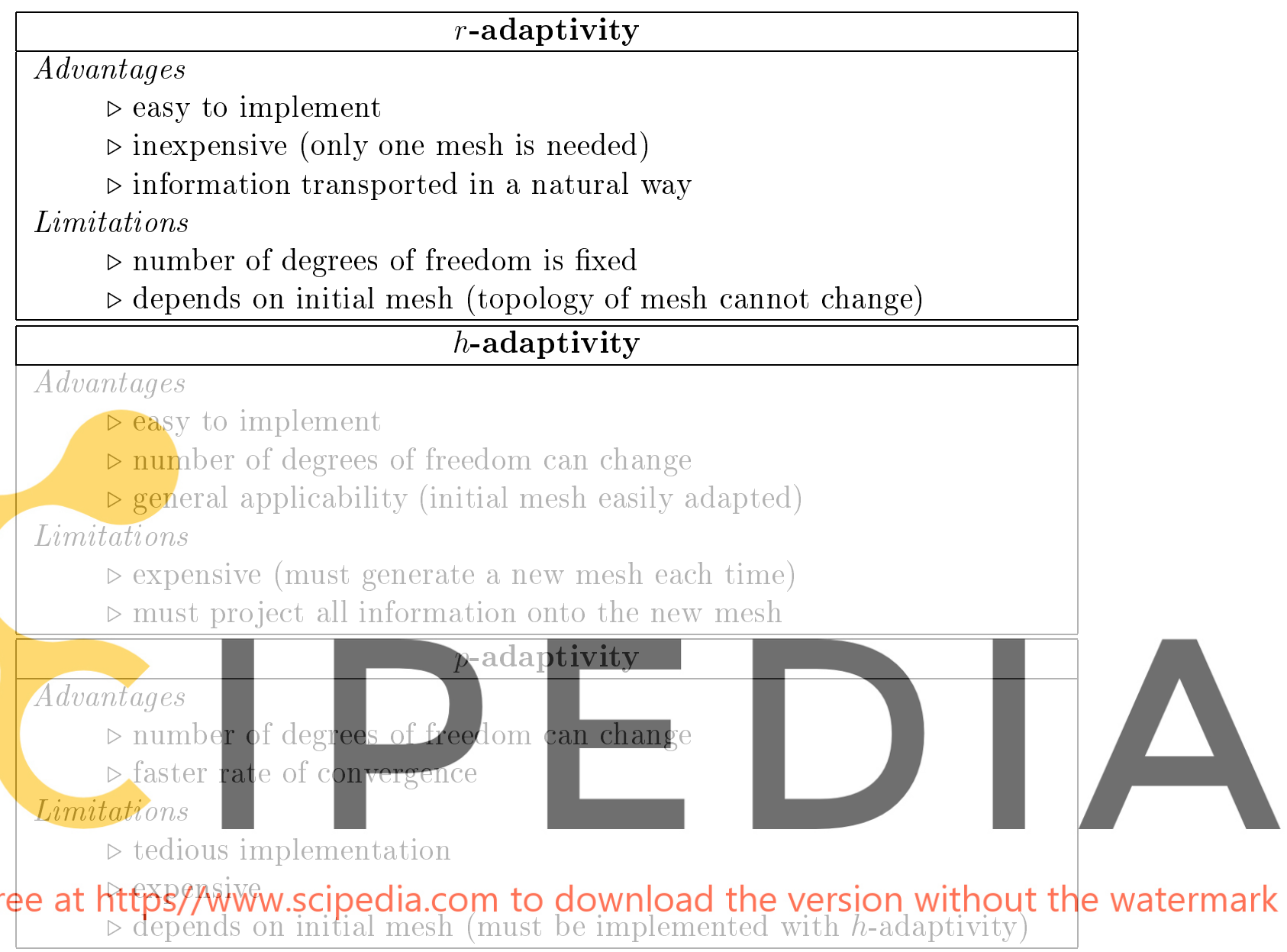

Table 2: Comparison of $r, h$ and $p$-adaptivity

\subsection{Adaptive strategies based on error assessment}

In principle, any of the approaches for error assessment (Table 1) can be combined with any of the procedures for adapting the spatial discretization (Table 2) to produce an adaptive strategy for finite element computations. However, some combinations are clearly to be preferred, as illustrated in Table 3.

Combining $r$-adaptivity and an error indicator provides a very simple adaptive strategy. As mentioned previously, an error indicator only gives relative information about the error. This is clearly a disadvantage of error 
indicators with respect to error estimators. However, relative information (i.e. where the error is larger and where it is smaller) is exactly what is needed for relocating the nodes. Since no new degrees of freedom can be added in $r$-adaptivity, the error indicator is used to decide where to put the available nodes. By doing so, an "optimal" use is made of the given mesh. Of course, the global accuracy of the solution cannot be prescribed a priori, because the number of degrees of freedom is fixed.

The conjunction of $h / p$-adaptivity and error estimation is also a valid strategy. In fact, it is the only approach that allows to obtain a solution with an (objective) accuracy prescribed a priori. The price to pay is a high computational cost. Note however that an extra ingredient is needed: an optimality criterion that relates the error at each point of the domain with the new element size or degree of the polynomial [28].

The combination of $r$-adaptivity and error estimation is clearly not an adequate strategy. All the effort in estimating the error in a quantitative and objective manner is wasted, because the information obtained cannot be fully exploited. In fact, using an error estimator to relocate the nodes overkills the problem, because only relative information can be accounted for
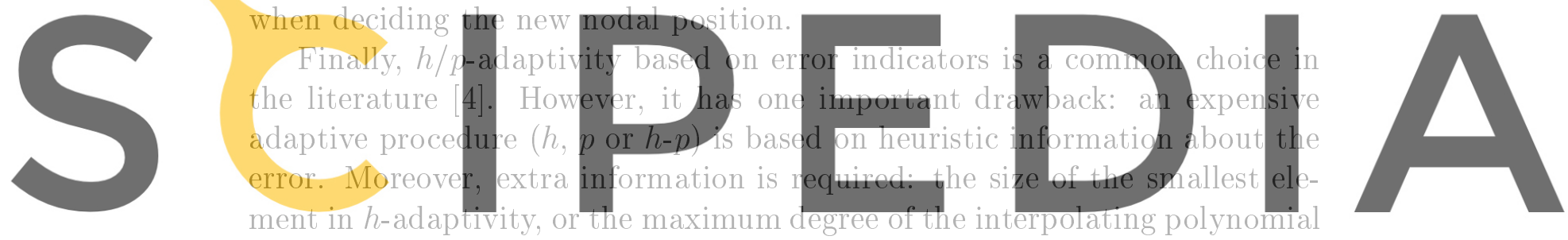

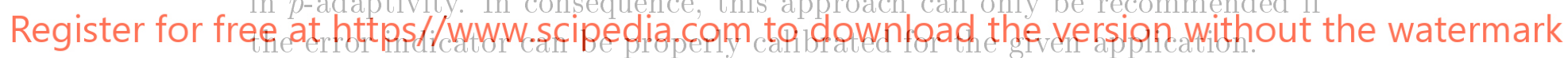

\begin{tabular}{|c|c|c|}
\hline & error indicator & error estimator \\
\hline$r$-adaptivity (ALE) & $\oplus$ & $\bigodot$ \\
$h / p$-adaptivity & $\bigodot$ & $\bigodot$ \\
\hline
\end{tabular}

Table 3: A global rating of adaptive strategies

In conclusion, the two best approaches consist on combining either simple ingredients ( $r$-adaptivity and error indicators) or more sophisticated ingredients $(h / p$-adaptivity and error estimators). The capabilities of these two 
strategies will be highlighted in the rest of the paper by means of some numerical examples. With the other two combinations, there is a clear unbalance between the tool used for assessing the error and the tool for adapting the spatial discretization.

\section{$3 \quad r$-adaptivity based on error indicators}

$r$-adaptivity based on error indicators is employed here for the prediction of yield line patterns in plates [29, 30]. Figure 2 shows a simply supported rectangular plate, with an eccentric hole and $5 \mathrm{~cm}$ thickness. The plate is subjected to a uniform load of $125 \mathrm{kN} / \mathrm{m}^{2}$. A bilinear elastoplastic behaviour is assumed, with Young's modulus $E=2 \cdot 10^{8} \mathrm{kN} / \mathrm{m}^{2}$, Poisson's ratio $\nu=0.2$, initial yield stress $\bar{\sigma}_{0}=2 \cdot 10^{5} \mathrm{kN} / \mathrm{m}^{2}$, and hardening modulus $h=E / 200$.

If a finite element analysis is performed on a fixed mesh, the results of Figure 3(a) are obtained. Due to the coarseness of the mesh, the spatial discretization is too poor and the yield line pattern (unknown a priori) is not properly captured. Of course, the solution can be improved by using a much finer mesh, see Figure 3(b). However, the computational cost is too high,
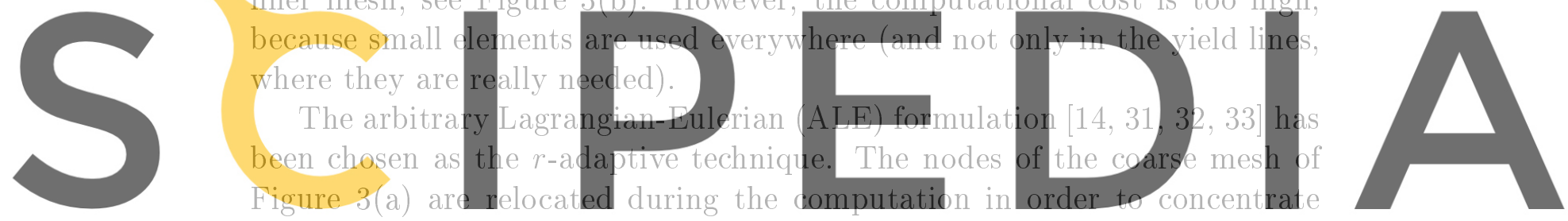

them along the yield lines. An error indicator is used for selecting the new

Register for free atl hetgisł $\phi$ www.scipedia.com to download the version without the watermark

For this particular application, the level of plastification provides a good indication of error $[29,30]$. The level of plastification is proportional to 1 ) the ratio of equivalent stress to the initial yield stress in elastic zones and to 2 ) the current yield stress in plastic zones. With this definition of the error indicator, the mesh is adapted in zones where yielding is taking place, and in zones that are about to yield.

It is important to note that this error indicator has the advantages and limitations stated in Table 1. It is based on a very intuitive assumption (error is larger where the nonlinearity -that is, plastification- is more important) and is simple to compute (because yield stresses are already computed in an analysis with a fixed mesh). However, the actual error of the solution is not quantified, and the indicator cannot be extended to other applications in a straightforward manner.

After the error indicator is computed, a criterion is needed for node relocation. Element size times the error indicator is prescribed to be constant 
for all the elements. This means that small elements are needed where the error indicator is large, and vice versa. With this criterion, new nodal coordinates are obtained by solving a diffusion equation [34]. When the nodes are relocated, the information must be transported from the old mesh to the new mesh. Since the two meshes share the same topology, this can be done by solving a convection equation. Here a Godunov-like technique is chosen $[35,17]$.

With the adaptive strategy just discussed, the solution of Figure 3(c) is obtained. The same coarse mesh of Figure 3(a) is chosen at the beginning of the analysis. Instead of keeping it fixed, however, the mesh is continuously adapted during the computation, by relocating the nodes after each load step. This enables a correct description of the yield line pattern (cf. Figures 3(b) and $3(\mathrm{c}))$.

A more quantitative comparison of the three solutions (coarse and fine fixed meshes, coarse adaptive mesh) is offered by Figure 4. Thanks to the node relocation, the profile of the equivalent plastic strain along the yield line with the coarse adaptive mesh is properly described. With the coarse fixed mesh, on the contrary, it is significantly underestimated.
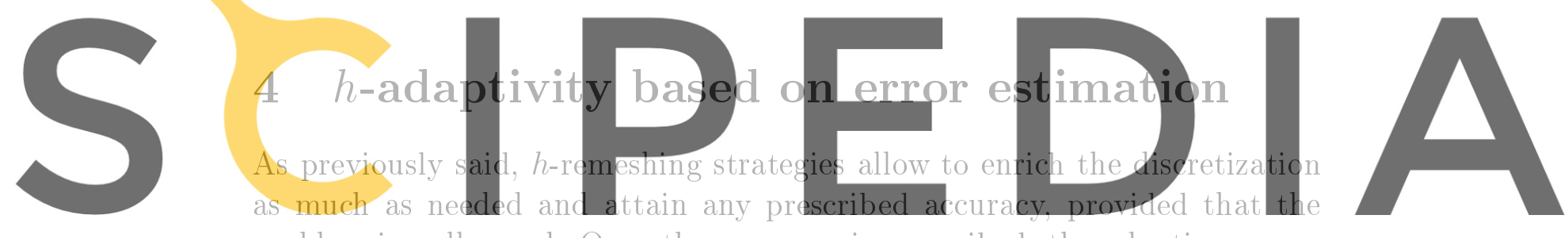

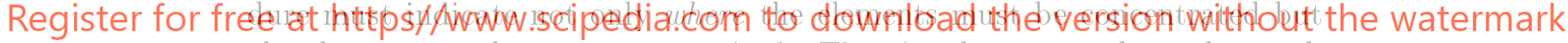
also how many elements are required. That is, the zones where the mesh must be refined have to be identified, and the required size of the mesh in every zone of the domain must be specified. Consequently, error estimators are required to obtain reliable information concerning the quantity of error and not only about its relative distribution.

The $h$-adaptivity processes consist on an iterative loop. For some mesh, the approximate solution is computed and the error is estimated. If the error is too large, the solution is considered unacceptable and the error distribution is used to define the element size for a new mesh in every zone of the domain. The new mesh is built up verifying the size prescriptions and the computations are re-started using this new mesh.

Two basic ingredients of $h$-adaptive procedures are the acceptability criterion and the remeshing strategy. The acceptability criterion is used as an stopping criterion for the iterative procedure. The remeshing strategy is the tool that allows to compute the prescribed element size from the estimated 
error distribution. Although the goal of the remeshing strategy is to provide a mesh that gives a solution verifying the acceptability criterion, the derivation of the remeshing strategies requires some additional assumptions, see [28]. These additional assumptions are called optimality criteria because they state the optimality properties of a finite element mesh by prescribing some uniformity of the error. Different optimality criteria lead to different remeshing strategies even if the acceptability criterion is the same.

The following examples of application of $h$-adaptivity to finite element computations are presented to illustrate three different topics.

1. The example introduced in section 4.1 shows the crucial role of the remeshing strategies in the resulting optimal mesh: under the same acceptability criterion but using remeshing strategies with different underlying optimality criteria, the adaptive process yields very different meshes.

2. The example introduced in section 4.2 stresses the importance of using a suitable error estimation tool. The error assessment must be able to account for all the sources of error. This is especially important in the case of shell problems where the discretization errors affect both the approximation of the geometry and the functional approximation.

3. Section 4.3 shows that in some problems even the general aspect of the mesh cannot be predicted a priori. The example analyzes the behavior of a plane strain compression specimen exhibiting strain localization. In this case the collapse mechanism is quite surprising. Consequently, the obtained solution is not intuitive and the optimal mesh is not trivial. The collapse mechanism is captured by a refined mesh but it cannot be predicted by a first guess of a mesh.

In all the examples the error is estimated using the technique introduced in $[18,24,25]$ and the quadrilateral meshes are generated following [36].

\subsection{Comparison of different remeshing strategies}

A 2-D plane strain analysis of a dam is presented, see Figure 5. This example is a benchmark test since it was introduced by Zienkiewicz and Zhu, see [6, 7]. In the adapted meshes, elements are concentrated around the corners of the semi-circular cavity, where the solution is singular. However, the number of elements and the mesh density are very different from one remeshing strategy to another.

Here three remeshing strategies found in the literature are used. 
1. The underlying optimality criterion used in the Li and Bettess (LB) remeshing strategy $[37,38]$ states that, in the optimal mesh, elementary errors are equal. The LB remeshing strategy has been proved to be optimal in the sense that yields meshes that minimize the number of elements for a given acceptability criterion.

2. The Zienkiewicz and Zhu (ZZ) remeshing strategy introduced in [6] is based in a simplification of the optimality criterion used by Li and Bettess.

3. The Oñate and Bugeda (OB) remeshing strategy is introduced in [39] and implements an optimality criterion based on engineering considerations. The mesh is assumed to be optimal if the density of error (ratio of the squared error norm over every element and its measure) is constant over the whole mesh.

For each one of these remeshing strategies an adaptive computation is carried out. The final meshes yielding acceptable solutions are shown in Figure 5. The number of required remeshing steps, the number of elements in the final mesh and the attained error are shown in Table 4. The distribution of elements in the final meshes yield by the LB and ZZ remeshing strategies are similar because the underlying optimality criteria are inspired by the same idea. On the other hand, the OB remeshing strategy leads to a very dense mesh (with more than five times the number of elements of the LB or ZZ strategies). This reveals that the condition imposed in the optimality criterion used in the OB strategy is much more demanding.

\begin{tabular}{|c|c|c|c|}
\hline Remeshing strategy & num. steps & num. elts. & \% error \\
\hline \hline original mesh & - & 193 & $11.48 \%$ \\
\hline LB & 2 & 685 & $4.62 \%$ \\
\hline ZZ & 4 & 602 & $4.93 \%$ \\
\hline OB & 3 & 3885 & $4.55 \%$ \\
\hline
\end{tabular}

Table 4: Summary of the results for the plane strain dam

\section{$4.2 \quad h$-adaptivity for shell problems}

The semispherical dome of Figure 6 is computed using thin shell elements. The structure is loaded by a vertical force uniformly distributed in the upper 
ring. The supports are distributed in the base as shown in Figure 6. This example is presented in reference [40]. The mechanical behavior is described by the deformed shape amplified $5 \times 10^{4}$ times, see Figure 7 and the Von Mises stresses distribution shown in Figure 8. In this example and in the remainder, the LB remeshing strategy is used because of its optimality from the viewpoint of computational economy. The sequence of meshes leading to a solution with a $5 \%$ of error is shown in Figure 9. Notice that, as expected and found by other authors [40], the elements are concentrated in the zones of the boundaries.

In standard plane or 3-D elasticity the discretization of the geometry affects only the boundary. On the contrary, in shell problems, this affects the whole domain. In fact for the first mesh (mesh 0 in Figure 9 ) the influence of the actual geometry in the error estimation is significant: the error estimate varies in the order of $10 \%$ depending on whether the actual geometry is accounted for or not. This source of errors may also be assessed if residual type error estimators are used. Flux projection error estimators are based on the analysis of discontinuities in the finite element approximate solution and, consequently, they do not use any information on the actual geometry. That means that, in shell analysis, standard flux projection error estimators are not able to take into account the effects of the geometry discretization in the computational error.

\subsection{Simulation of strain localization via adaptive remeshing}

This example is used to show the ability of adaptive strategies to capture unexpected solutions (complex failure mechanisms) [41, 42]. The example reproduces the compression of a plane strain rectangular specimen. The test is driven by imposing the velocity at the top of the specimen. In order to induce the strain localization, circular openings are introduced, playing the role of imperfections. In this case, the specimen has two circular openings symmetric with respect to the center. That allows to study only one half of the domain, see Figure 10.

Figure 11 shows the sequence of meshes in this case. It is worth noting that, in the final mesh, according to the concentration of elements, two bands are developed. In fact, the resulting bands are not aligned with the imperfections, as it could be expected, but have an opposite inclination. Meshes 0 and 1 are not able to reproduce the behaviour of the actual (final) solution

because the elements in the zone of the second band (which develops in a further stage of the loading process) are too large and, consequently, the dis- 
cretization is too stiff. Then, the size of the elements in this zone does not allow the inception of softening. However, the error estimator indicates that the elements must be reduced in the zone of the second band. Thus, once the remeshing process introduces small enough elements along the second band, in meshes 2 to 5 , a second mechanism can also be captured. Figure 11 shows also the distribution of the error along the remeshing process, which tends to be uniform, as expected. In the first meshes, the error is larger along the bands and, consequently, the successive discretizations concentrate elements in these zones. Notice that the elements are, in fact, concentrated along the edges of the bands, where the gradients of the displacements are large.

The evolution of the meshes in the remeshing sequence of Figure 11 suggest that the actual complex failure mechanism is ignored by the first discretizations and can only be captured using the adapted meshes. This is confirmed comparing the deformation patterns and the force-displacement curves obtained with different meshes.

Figure 12 shows how the computed equivalent inelastic strain and the deformation evolve along the remeshing process. Only after two remeshing steps the mesh captures two bands. In the previous meshes the discretization is not accurate enough and only one band is completely developed. Since large deformations are considered, once the first band evolves enough, the kinematic mechanism associated with this band locks. Then a second band appears as a new deformation mode with less energy. Figure 12 shows also how the force-displacement curves for meshes 0 and 1 are qualitatively different from those of meshes 2 to 5 . In fact the shapes of the force-displacement curves for meshes 2, 3, 4 and 5 are practically identical and have two inflections in the descending branch. The solution given by the last mesh is obviously more accurate than the original one because the energy of deformation (area under the force-displacement curve) is lower. In fact, since the error is controlled in energy norm, one can be sure that the actual curve, associated with the exact solution, is not too far from the obtained curve (the error in energy norm is less than $1.5 \%$ and, consequently the difference of the area under the curves is less than $1.5 \%$ ).

Thus, this example demonstrates that adaptivity based on error estimation is an essential tool for the determination of a priori unpredictable final solutions. Without this adaptive strategy, the initial mesh (mesh 0 in Figure 11) and the resulting solution could be regarded as correct, and the second mechanism would not be detected. 


\section{Concluding remarks}

The merits and limitations of various adaptive finite element strategies have been discussed. First, the two basic ingredients -namely, a tool for error assessment and a procedure for adapting the spatial discretization- have been analyzed separately. After that, various combinations of these techniques have been assessed.

The best approaches consist on combining ingredients of similar complexity. If $r$-adaptivity is combined with error indicators, a very simple and computationally efficient adaptive strategy is obtained. The conjunction of $h / p$-adaptivity and error estimators, on the other hand, results in a more sophisticated and costly strategy, which allows to obtain a solution with a prescribed accuracy.

The other two combinations (r-adaptivity with error estimators, or $h / p$ adaptivity with error indicators) are less attractive, because there is a clear unbalance between the tools combined.

The two adequate strategies have been illustrated by means of several numerical examples in linear and nonlinear solid mechanics.

\section{References}

[1] Babuška, I., T. Strouboulis, C.S. Upadhyay and K.S. Gangaraj (1995), "A posteriori estimation and adaptive control of the pollution error in the $h$-version of the finite element method", International Journal for Numerical Methods in Engineering, 38, 4207-4235.

[2] Babuška, I., T. Strouboulis, K.S. Gangaraj and C.S. Upadhyay (1997), "Pollution error in the $h$-version of the finite element method and the local quality of the recovered derivatives", Computer Methods in Applied Mechanics and Engineering, 140, 1-37.

[3] Babuška, I., T. Strouboulis and K.S. Gangaraj (1997), "A posteriori estimation of the error in the recovered derivatives of the finite element solution", Computer Methods in Applied Mechanics and Engineering, 150, 369-396.

[4] Belytschko, T. and M. Tabbara (1993), " $h$-adaptive finite element methods for dynamic problems, with emphasis on localization", International Journal for Numerical Methods in Engineering, 36, 4245-4265. 
[5] Peraire, J., M. Vahdati, K. Morgan and O.C. Zienkiewicz (1991), "Adaptive remeshing for compressible flow computations", Journal of Computational Physics, 72, 449-466.

[6] Zienkiewicz, O.C. and J.Z. Zhu (1987), "A simple error estimator and adaptive procedure for practical engineering analysis", International Journal for Numerical Methods in Engineering, 24, 337-357.

[7] Zienkiewicz, O.C. and J.Z. Zhu (1992), "The superconvergent patch recovery (SPR) and adaptive finite element refinement", Computer Methods in Applied Mechanics and Engineering, 101, 207-224.

[8] Wiberg, N.E. and P. Hager (1998), "Error estimation and adaptivity for $h$-version eigenfrequency analysis", in P. Ladevèze and J.T. Oden eds, Advances in Adaptive Computational Methods in Mechanics, Elsevier, 461-476.

[9] Bouillard, Ph. and F. Ihlenburg (1998), "Error estimation and adaptivity for the finite element method in acoustics", in P. Ladevèze and J.T. Oden eds, Advances in Adaptive Computational Methods in Mechanics, Elsevier, 477-492.

[10] Sarrate, J., J. Peraire and A. Patera, "A posteriori finite element error bounds for nonlinear outputs of the Helmholtz equation", to appear in International Journal for Numerical Methods in Fluids.

[11] Basu, P.K. and A.G. Peano (1983), "Adaptivity in $p$-version finite element analysis", Journal of Structural Engineering, 109, 2310-2324.

[12] Ainsworth, M. and J.T. Oden (1992), "A procedure for a posteriori error estimation for $h-p$ finite element methods", Computer Methods in Applied Mechanics and Engineering, 101, 73-96.

[13] Ainsworth, M. and A. Arnold (1998), "A reliable a posteriori error estimator for adaptive hierarchic modelling", in P. Ladevèze and J.T. Oden eds, Advances in Adaptive Computational Methods in Mechanics, Elsevier, 101-114.

[14] Donea, J. (1983), "Arbitrary Lagrangian-Eulerian finite element methods", Chapter 10 of Computational Methods for Transient Analysis, Eds. T. Belytschko and T.J.R. Hughes, Elsevier, New York, USA.

[15] Miller, M.J. (1994), Moving finite elements, Clarendon Press, Oxford. 
[16] Simo, J.C. (1988), "A framework for finite strain elastoplasticity based on maximum plastic dissipation and the multiplicative decomposition. Part II: computational aspects", Computer Methods in Applied Mechanics and Engineering, 68, 1-31.

[17] Rodríguez-Ferran, A., F. Casadei and A. Huerta, "ALE stress update for transient and quasistatic processes", International Journal for $\mathrm{Nu}$ merical Methods in Engineering, in press.

[18] Huerta, A. and P. Díez, "Error estimation including pollution assessment for nonlinear finite element analysis", submitted to Computer Methods in Applied Mechanics and Engineering.

[19] Babuška, I. and C. Rheinboldt (1978), "A-posteriori error estimates for the finite element method", International Journal for Numerical Methods in Engineering, 12, 1597-1615.

[20] Bank, R.E. and A. Weiser (1985), "Some a posteriori error estimators for elliptic partial differential equations", Mathematics of Computation, 44, 283-301.

[21] Ladevèze, P., J.P. Pelle and Ph. Rougeot (1991), "Error estimation and mesh optimization for classical finite elements", Engineering Computations, 8, 69-80.

[22] Ainsworth, M. and J.T. Oden (1993), "A unified approach to a posteriori error estimation using element residual methods", Numerische Mathematik, 65, 23-50.

[23] Díez, P., J.J. Egozcue and A. Huerta (1998), "Analysis of the average efficiency of an error estimator", in M. Kř́ižek et al. eds, Finite Element Methods: Superconvergence, Post-processing and a Posteriori Error Estimates, Marcel Dekker, New York, 113-126.

[24] Huerta, A., P. Díez and J.J. Egozcue (1998), "Error estimation for linear and nonlinear problems", in M. Křížek et al. eds, Finite Element Methods: Superconvergence, Post-processing and a Posteriori Error Estimates, Marcel Dekker, New York, 183-194.

[25] Díez, P., J.J. Egozcue and A. Huerta, "A posteriori error estimation for standard finite element analysis", to appear in Computer Methods in Applied Mechanics and Engineering. 
[26] Giuliani, S. (1982), "An algorithm for continuous rezoning of the hydrodynamic grid in Arbitrary Lagrangian-Eulerian computer codes", $\mathrm{Nu}$ clear Engineering and Design, 72, 205-212.

[27] Oden, J.T. and S. Prudhomme (1998), "A technique for a posteriori error estimation of $h-p$ approximations of the Stokes problem", in P. Ladevèze and J.T. Oden eds, Advances in Adaptive Computational Methods in Mechanics, Elsevier, 43-64.

[28] Díez, P. and A. Huerta, "A unified approach to remeshing strategies for finite element $h$-adaptivity", submitted to Computer Methods in Applied Mechanics and Engineering.

[29] Askes, H., A. Rodríguez-Ferran and A. Huerta, "Adaptive analysis of yield line patterns in plates with the Arbitrary Lagrangian-Eulerian method", accepted for publication in Computers and Structures.

[30] Rodríguez-Ferran, A., H. Askes and A. Huerta (1998), "Arbitrary Lagrangian-Eulerian analyses of plastic failure in plates", Fourth World Congress on Computational Mechanics, Buenos Aires, Argentina.

[31] Huétink, J. (1986), On the simulation of thermo-mechanical forming processes, Ph.D. Dissertation, University of Twente, The Netherlands.

[32] Huerta, A. and W.K. Liu (1988), "Viscous flow with large free surface motion", Computer Methods in Applied Mechanics and Engineering, 69, $277-324$.

[33] Benson, D.J. (1989), "An efficient, accurate, simple ALE method for nonlinear finite element programs", Computer Methods in Applied Mechanics and Engineering, 72, 305-350.

[34] Pijaudier-Cabot, G., L. Bodé and A. Huerta (1995), “Arbitrary Lagrangian-Eulerian finite element analysis of strain localization in transient problems", International Journal for Numerical Methods in Engineering, 38, 4171-4191.

[35] Huerta, A. and F. Casadei (1994), "New ALE applications in non-linear fast-transient solid dynamics", Engineering Computations, 11, 317-345.

[36] Sarrate, J. (1996), Modelización numérica de la interacción fluido-sólido rígido: desarrollo de algoritmos, generación de mallas y adaptabilidad, Doctoral Thesis, Universitat Politècnica de Catalunya, Barcelona. 
[37] Li, L.Y., P. Bettess, J.W. Bull, T. Bond and I. Applegarth (1995), "Theoretical formulations for adaptive finite element computations", Communications in Numerical Methods in Engineering, 11, 857-868.

[38] Li, L.Y. and P. Bettess (1995), "Notes on mesh optimal criteria in adaptive finite element computations", Communications in Numerical Methods in Engineering, 11, 911-915.

[39] Oñate, E. and G. Bugeda (1993), "A study of mesh optimality criteria in adaptive finite element analysis", Engineering Computations, 10, 307321.

[40] Cirak, F. and E. Ramm, "A-posteriori error estimation and adaptivity for linear elasticity using the reciprocal theorem", submitted to Computer Methods in Applied Mechanics and Engineering.

[41] Huerta, A., P. Díez, A. Rodríguez-Ferran and G. Pijaudier-Cabot (1998), "Error estimation and adaptive finite element analysis of softening solids", in P. Ladevèze and J.T. Oden eds, Advances in Adaptive Computational Methods in Mechanics, Elsevier, 333-348.

[42] Díez, P., M. Arroyo and A. Huerta, "Adaptivity based on error estimation for viscoplastic softening materials", submitted to Mechanics of Cohesive-Frictional Materials. 


\section{List of Figures}

1 Necking test: (a) coarse fixed mesh, (b) $r$-adaptivity based on an error indicator, (c) $h$-adaptivity based on error estimation . 20

2 Rectangular plate with square hole . . . . . . . . . 21

3 Equivalent plastic strain: (a) coarse fixed mesh, (b) fine fixed mesh, (c) adaptive coarse mesh . . . . . . . . . . 22

4 Profiles of equivalent plastic strain along $y=x+3$. Fine fixed mesh (black), coarse fixed mesh (blue) and adaptive coarse mesh (red). ................... 23

5 Plane strain dam example: final mesh of the adaptive procedure using different remeshing strategies . . . . . . . . . 24

6 Semispherical dome: description of the geometry and the loading conditions . . . . . . . . . . . . . 25

7 Semispherical dome: deformed shape . . . . . . . . 26

8 Semispherical dome: Von Mises stress distribution . . . . . . . 27

9 Semispherical dome: sequence of meshes . . . . . . . . . 28

10 Rectangular specimen with two symmetric imperfections . . . 29

11 Remeshing process using Li-Bettess for a prescribed accuracy of $1.5 \%$ : sequence of meshes and estimated error distributions 30

12 Numerical bifurcation in the first meshes: mesh deformation amplified 40 times and equivalent inelastic strain contours . . 31

\section{List of Tables}

1 Comparison of error estimators and error indicators . . . . . 4

2 Comparison of $r, h$ and $p$-adaptivity . . . . . . . 6

3 A global rating of adaptive strategies . . . . . . . . . . . 7

4 Summary of the results for the plane strain dam . . . . . . . 11 


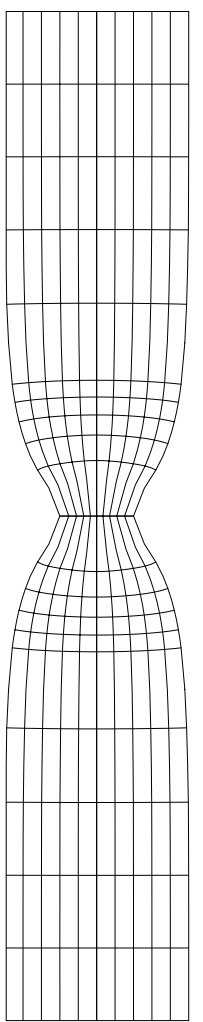

(a)

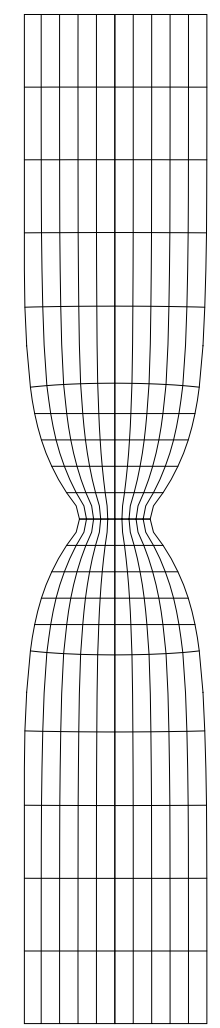

(b)

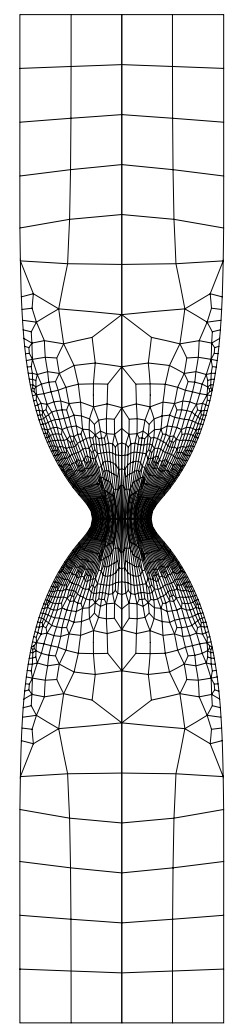

(c)

Figure 1: Necking test: (a) coarse fixed mesh, (b) $r$-adaptivity based on an error indicator, (c) $h$-adaptivity based on error estimation 


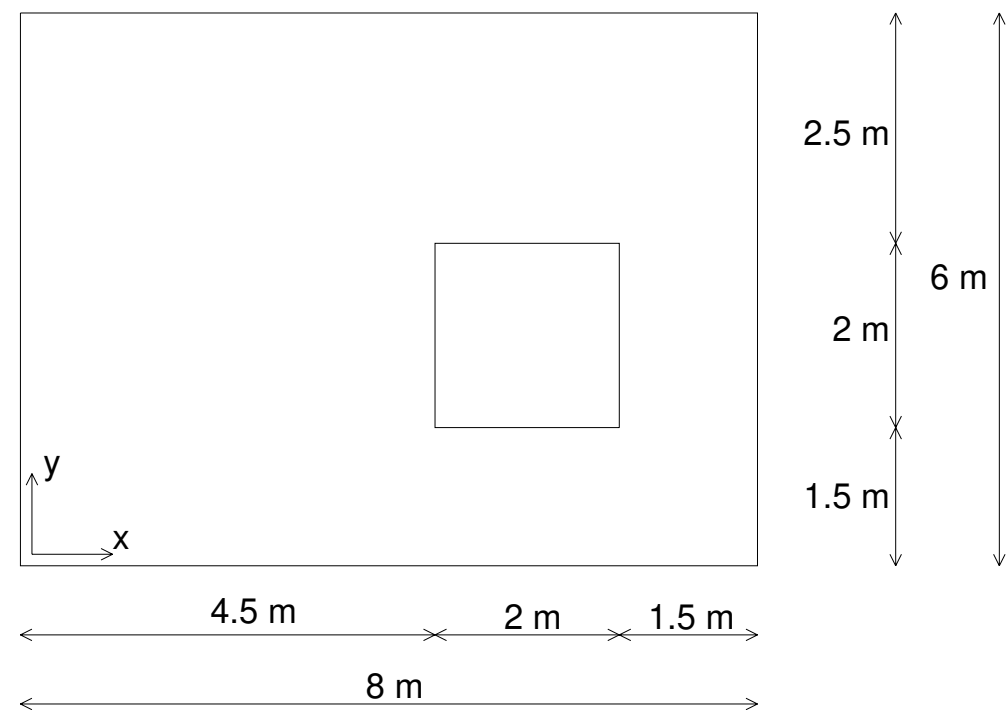

Figure 2: Rectangular plate with square hole 


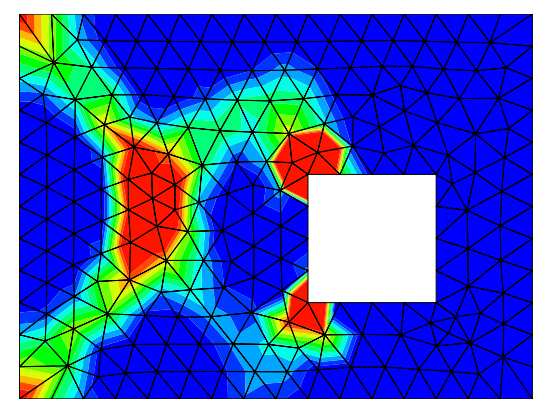

(a)

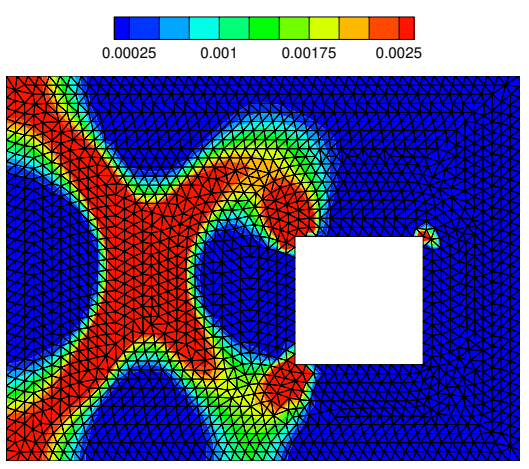

(b)

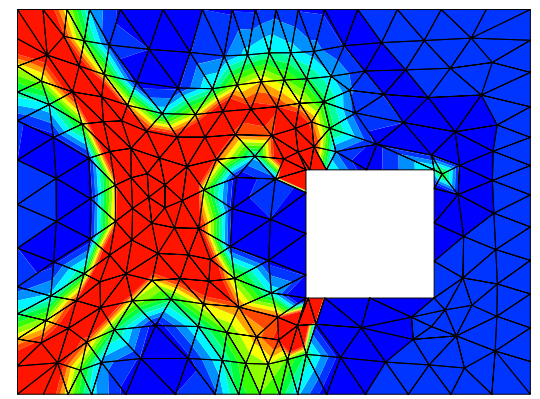

(c)

Figure 3: Equivalent plastic strain: (a) coarse fixed mesh, (b) fine fixed mesh, (c) adaptive coarse mesh 


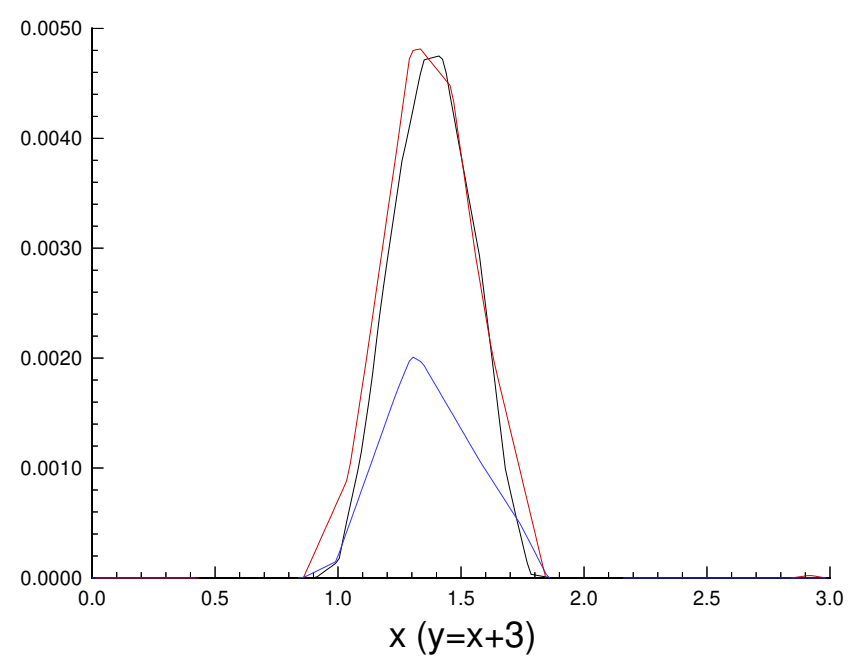

Figure 4: Profiles of equivalent plastic strain along $y=x+3$. Fine fixed mesh (black), coarse fixed mesh (blue) and adaptive coarse mesh (red). 

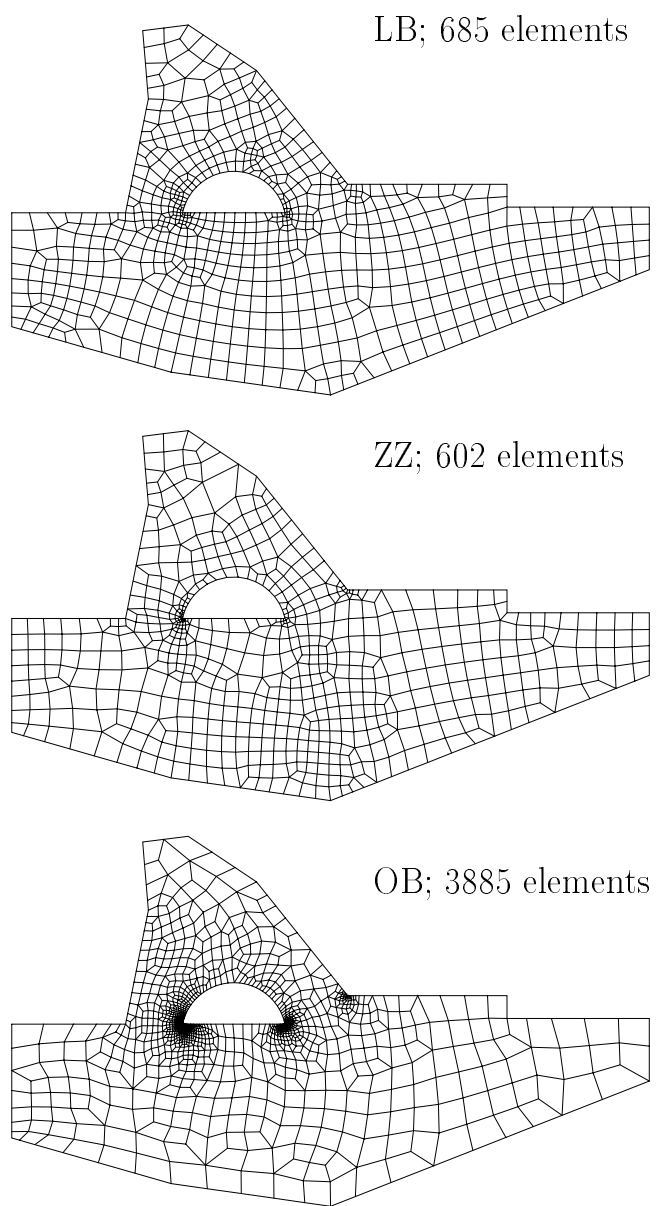

Figure 5: Plane strain dam example: final mesh of the adaptive procedure using different remeshing strategies 

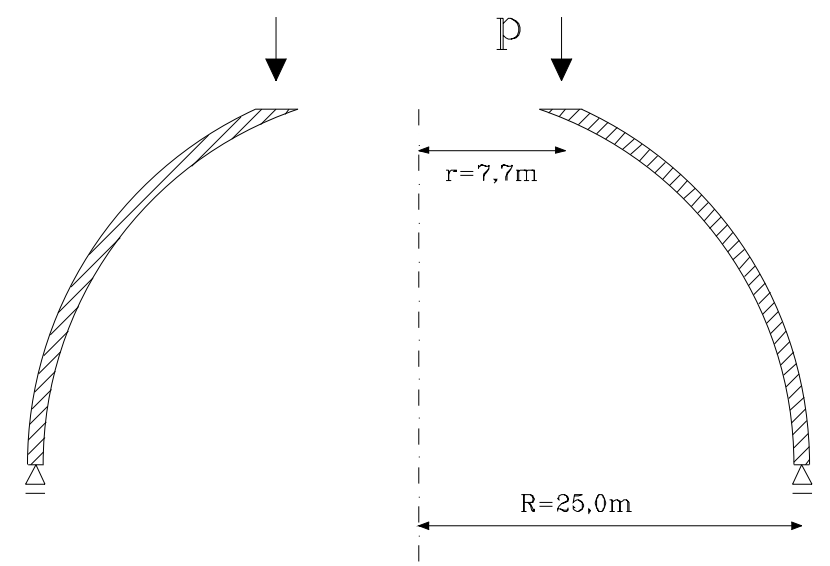

$$
\begin{aligned}
& \mathrm{E}=1,092 \mathrm{MPa} \\
& \mathrm{t}=1,0 \mathrm{~m} \\
& \mathrm{P}=1,0 \mathrm{~N} / \mathrm{m} \\
& \mathrm{V}=0,3
\end{aligned}
$$

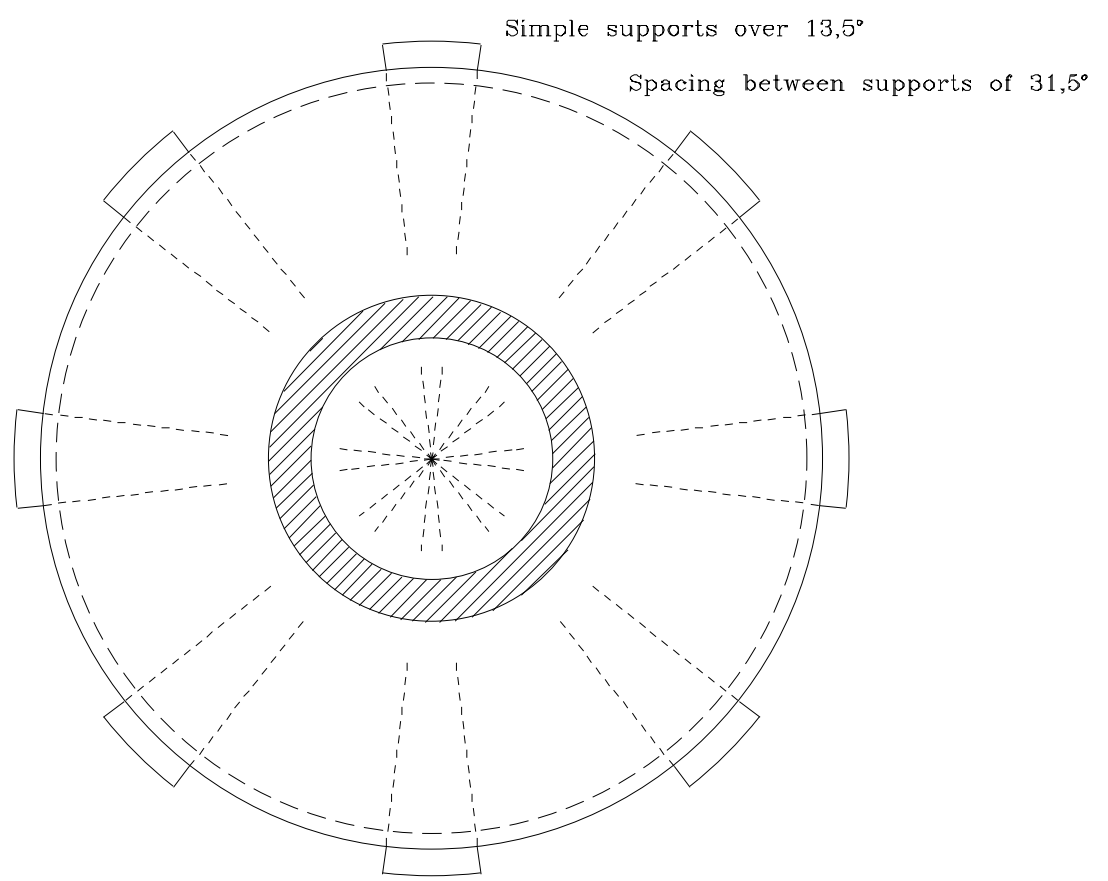

Figure 6: Semispherical dome: description of the geometry and the loading conditions 


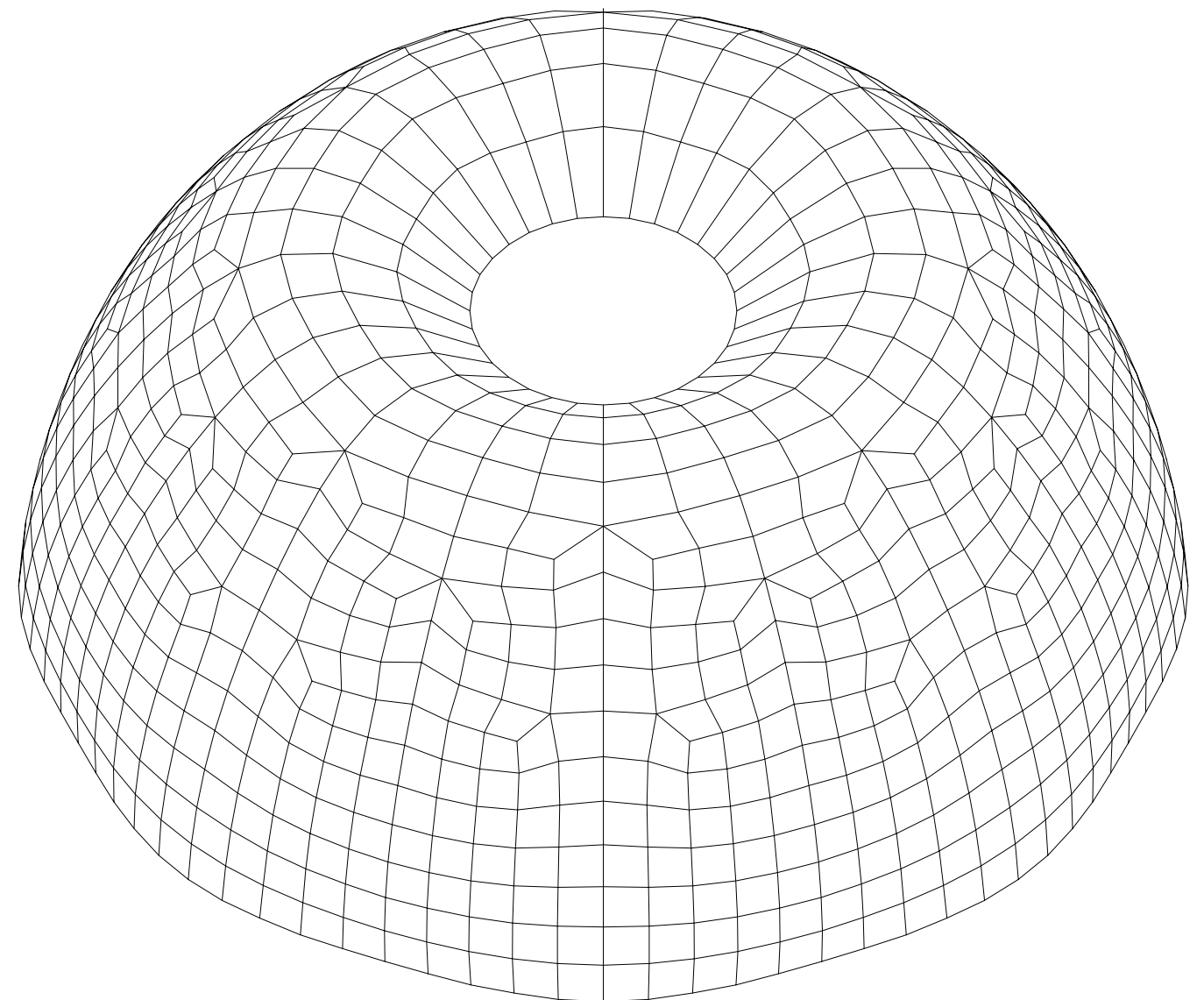

Figure 7: Semispherical dome: deformed shape 


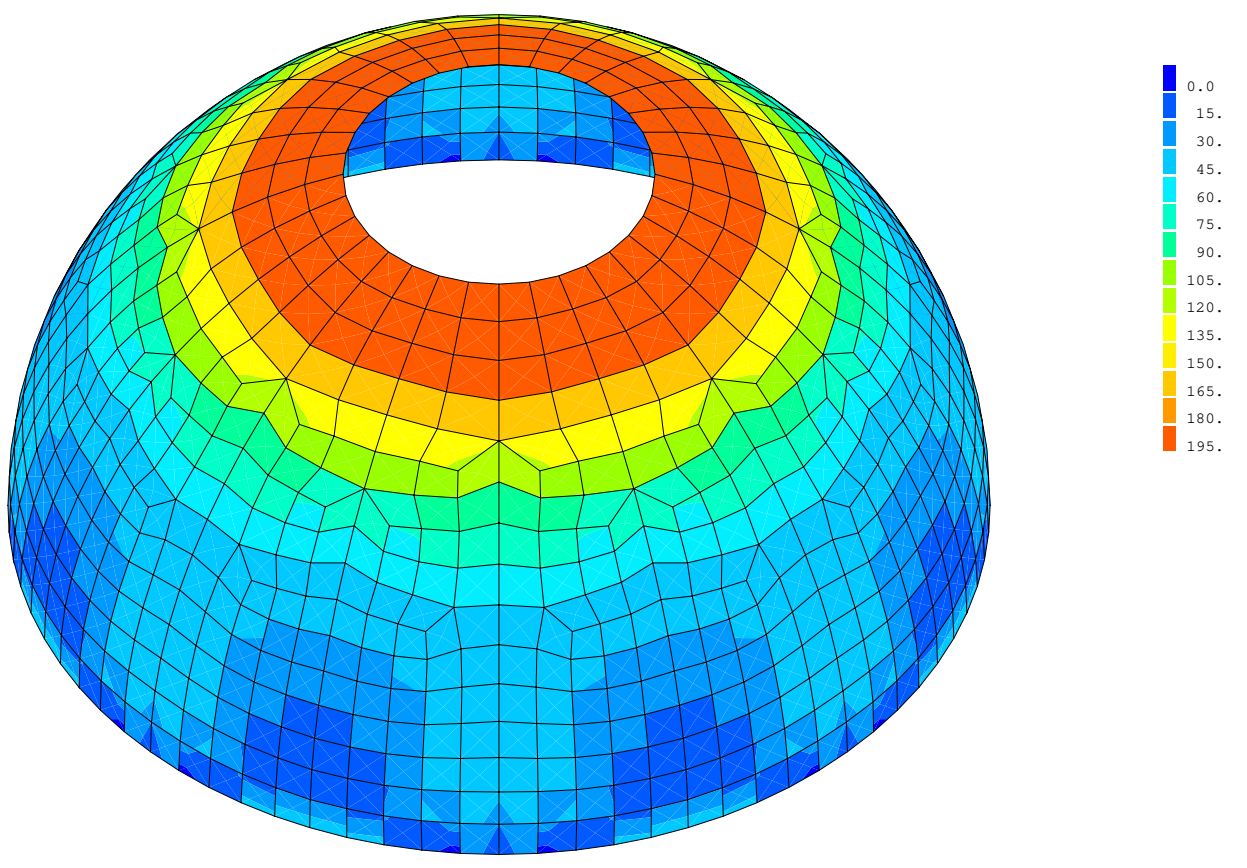

Figure 8: Semispherical dome: Von Mises stress distribution 

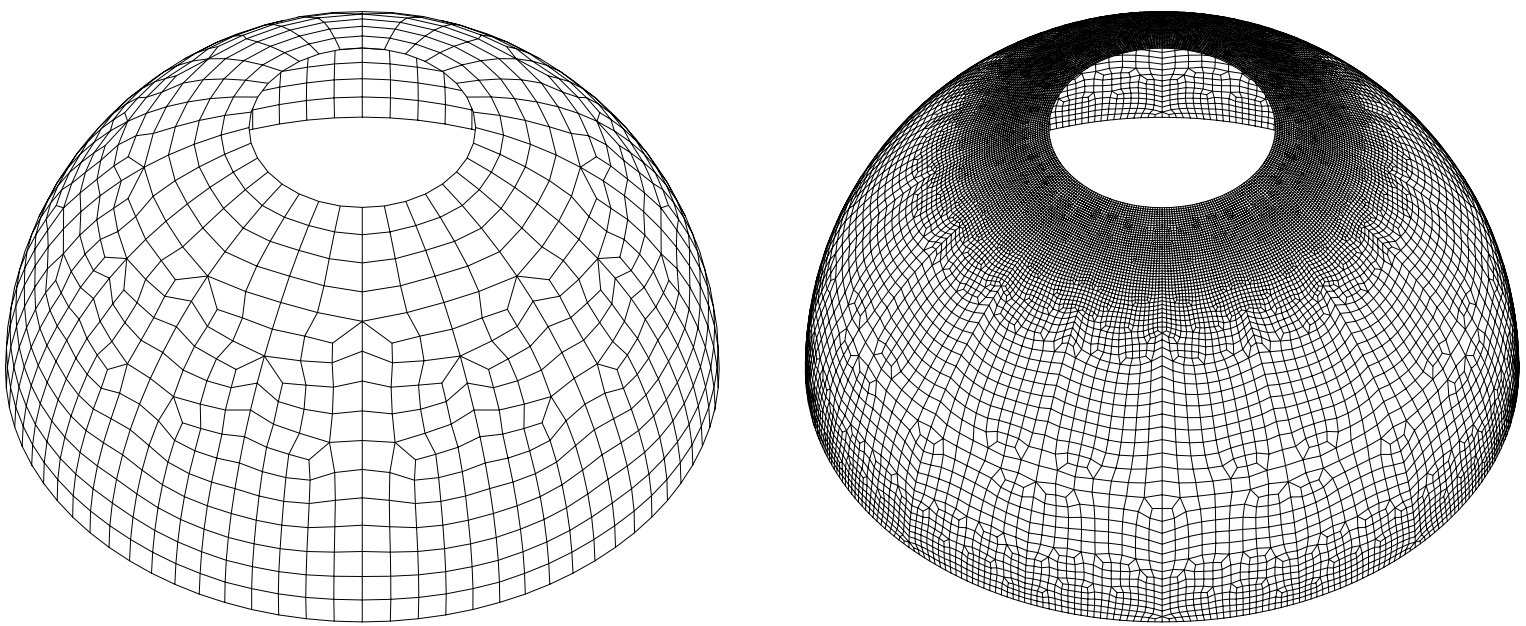

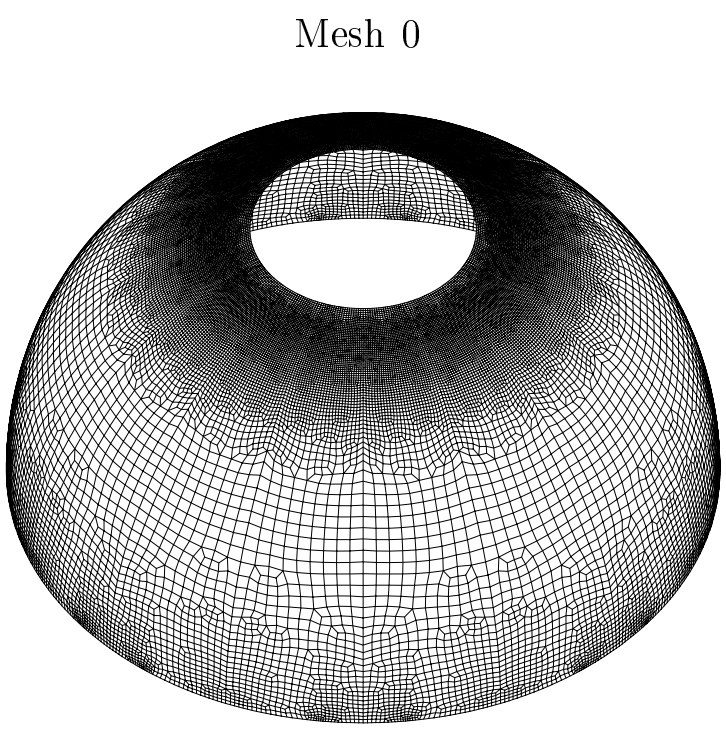

Mesh 2

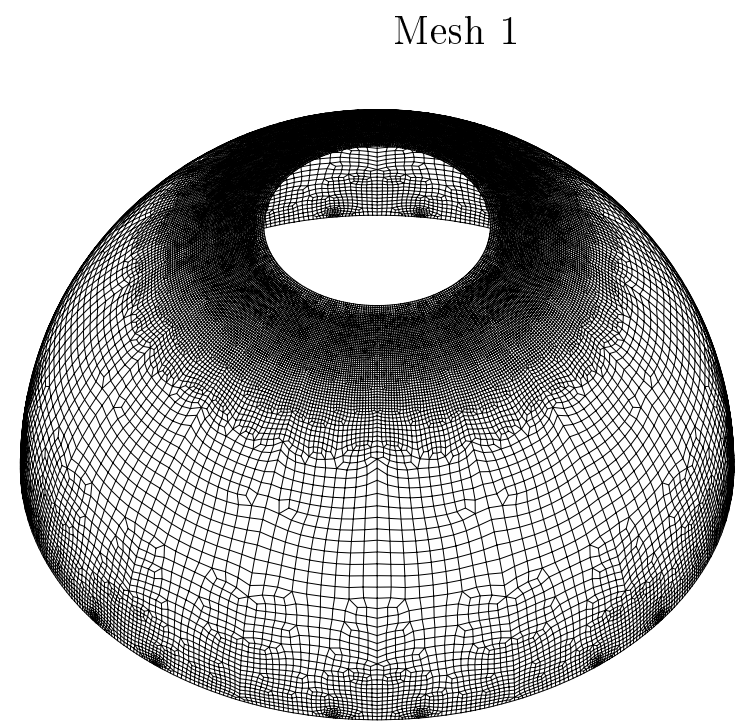

Mesh 3

Figure 9: Semispherical dome: sequence of meshes 


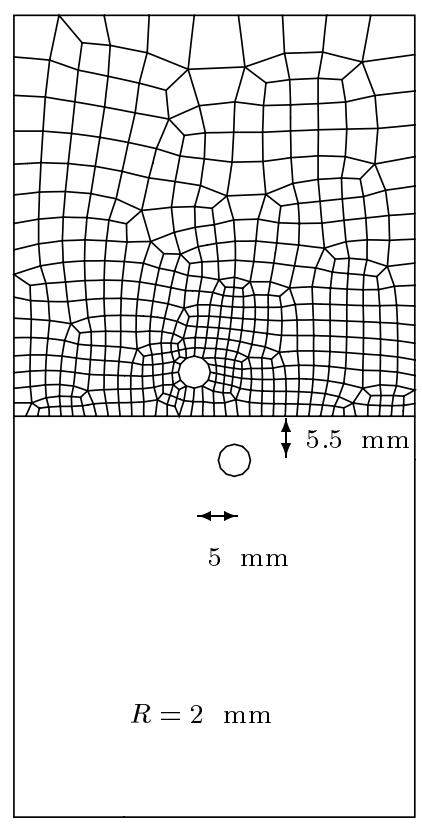

Figure 10: Rectangular specimen with two symmetric imperfections 


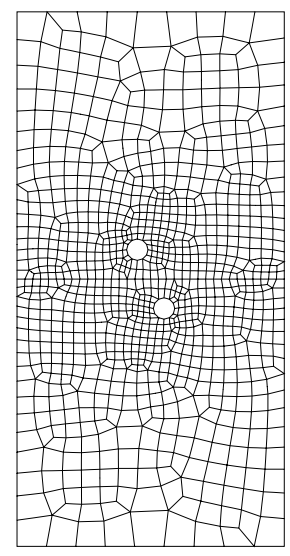

Mesh 0; 462 elements;

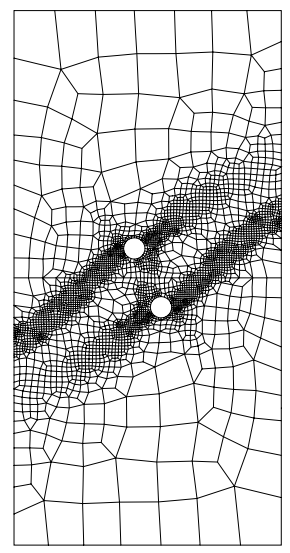

Mesh 2; 1641 elements;
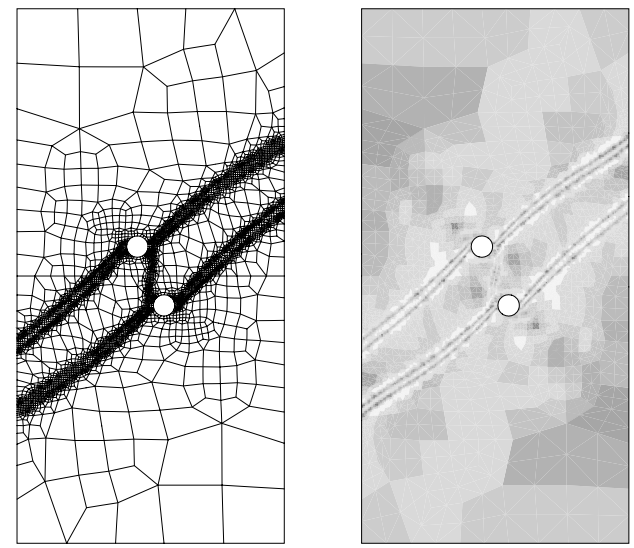

Mesh 4; 3200 elements; global accuracy: $1.73 \%$

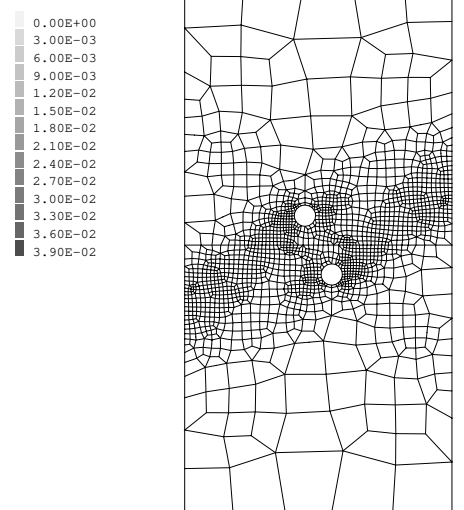

Mesh 1; 856 elements;

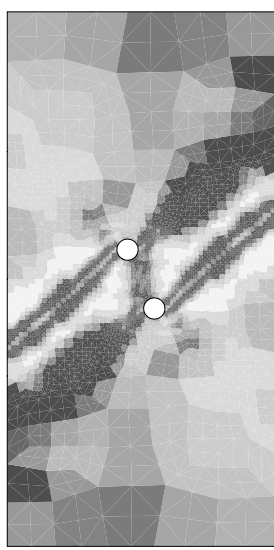

global accuracy: $4.00 \%$
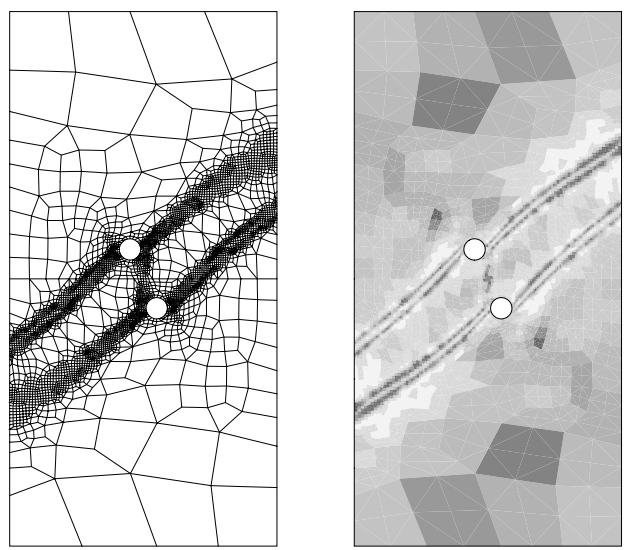

Mesh 3; 2235 elements;

global accuracy: $2.31 \%$
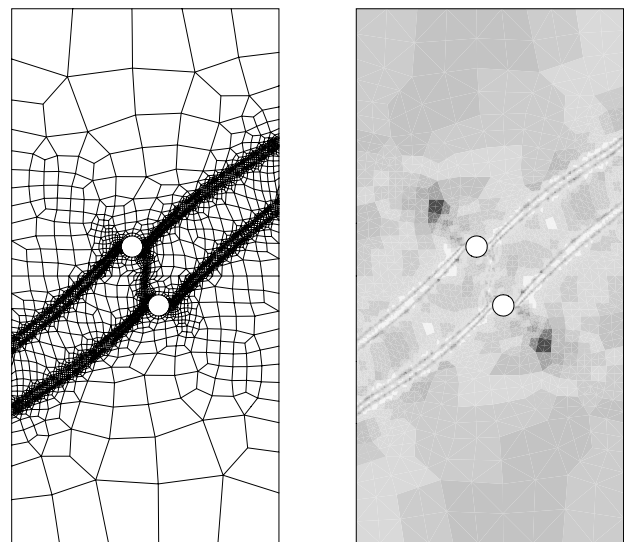

Mesh 5; 3307 elements; global accuracy: $1.49 \%$

Figure 11: Remeshing process using Li-Bettess for a prescribed accuracy of 1.5\%: sequence of meshes and estimated error distributions 


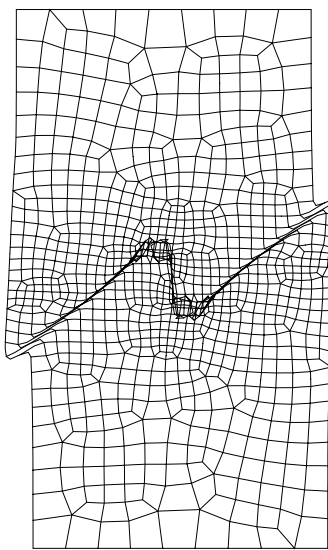

Mesh 0; two shear bands completely developed

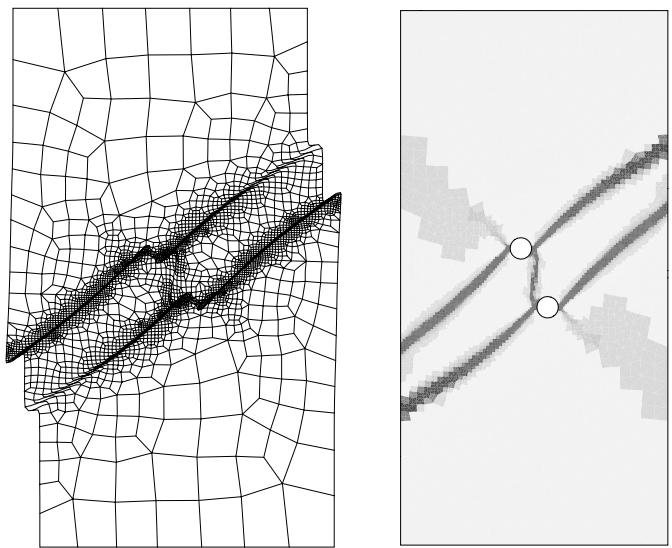

Mesh 2; four shear bands completely developed
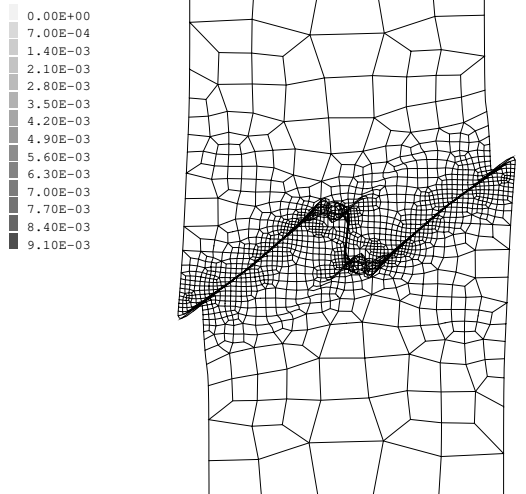

Mesh 1; two shear bands completely developed

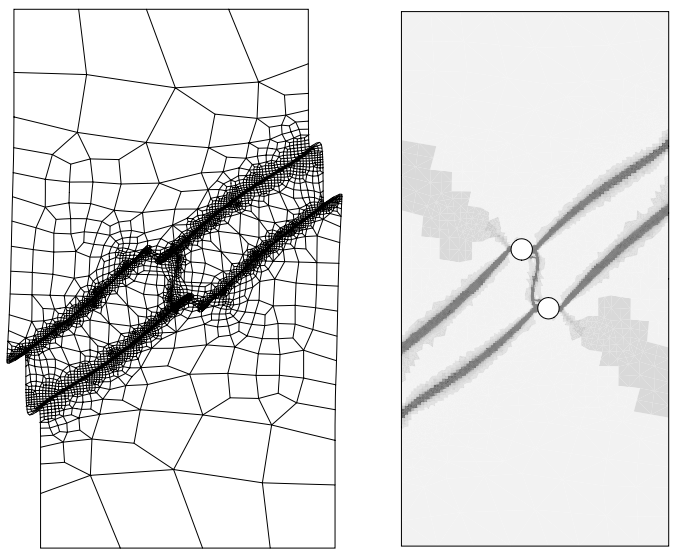

Mesh 3; four shear bands completely developed

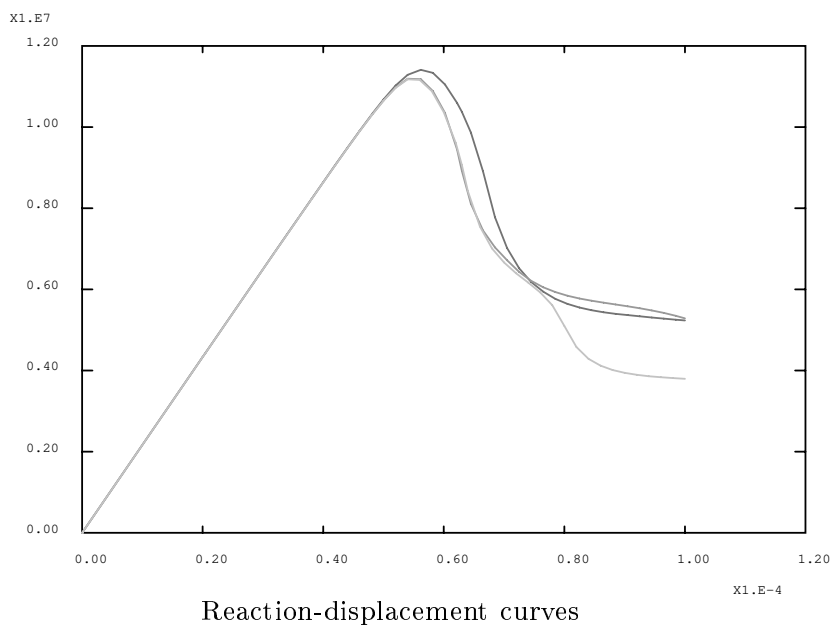

Dark curve: mesh 0

Medium curve: mesh 1

Bright curve: meshes 2 and 3

Figure 12: Numerical bifurcation in the first meshes: mesh deformation amplified 40 times and equivalent inelastic strain contours 